Pacific Journal of Mathematics

NOTE ON Fr.FеERECT MoDULe 


\title{
A NOTE ON FR-PERFECT MODULES
}

\author{
R. D. KeTKaR AND N. VANAJA
}

This paper defines and characterizes projective FR-perfect modules which is a generalization of semiperfect modules. Using these some characterizations of semiperfect modules are obtained.

Introduction. Let $R$ be a ring with identity. All modules we consider are unitary left $R$-modules. A submodule $N \subseteq M$ is said to be small in $M$ if $N+T=M$ implies $T=M$. An epimorphism $P \stackrel{f}{\rightarrow} M$ is called minimal if $\operatorname{Ker}(f)$ is small in $P$. A minimal epimorphism $P \stackrel{f}{\rightarrow} M$, where $P$ is projective, is called a projective cover of $M$. We denote by $J$, the Jacobson radical of $R$. By $J(M)$ we mean the radical (intersection of all maximal submodules) of a module $M$. If $M$ is projective $J(M)=J M$. We call a module $N$ $M$-finitely related $\left(M\right.$-FR) if $N \cong M^{n} / B$, where $B$ is finitely generated. A module $M$ is called FR-perfect if every $M$-FR module has a projective cover. Similarly we define finitely presented perfect (FRperfect) modules.

Our aim is to characterise $M$-FR perfect projective modules. So we would like to find out equivalent conditions for a module $M / A$ to have a projective cover, where $M$ is projective. In $\S 1$ we do this when either $A$ is finitely generated or $J M$ is small in $M$. In particular, we show that for a projective module $M, M / A$ has a projective cover if and only if $f(A)$ is a direct summand of $M / J M$ and any direct decomposition $f(A) \oplus B$ of $M / J M$ can be lifted up, where the summand of $M$ corresponding to $f(A)$ is finitely generated and $f: M \rightarrow M / J M$ is the natural projection. So for a projective FR-perfect module every finitely generated submodule of $M / J M$ is a direct summand. That is $M / J M$ is a regular $R / J$-module (R. Ware [8]). We prove some properties of regular modules which are used later in proving that direct sum of FR-perfect projective modules is FR-perfect projective if and only if each summand is so.

In $\S 2$, we give several characterizations of FR-perfect projective modules. We prove that the following conditions are equivalent for a projective $R$-module $M$ (i) $M$ is FR-perfect (ii) $M / J M$ is a regular $R / J$ module and any direct decomposition $A \oplus B$ of $M / J M$ can be lifted up whenever $A$ is cyclic (finitely generated) and the summand of $M$ corresponding to $A$ is finitely generated and (iii) $M / U$ has a projective cover whenever $U$ is cyclic (finitely generated). If further $J M$ is small in $M$ then the above conditions are equivalent to (iv) 
$M / J M$ is a regular $R / J$ module and every direct decomposition of $M / J M$ can be lifted up and $(v) M / J M$ is a regular $R / J$-module and every direct summand of $M / J M$ has a projective cover. Using these we give some characterizations of semiperfect ring. We prove that a projective module $M$ is semiperfect if and only if $J M$ is small in $M$ and $M / U$ has a projective cover whenever $U$ is countably generated. We also show that a module $M$ is semiperfect if and only if $M$ is FR-perfect projective, $J M$ is small in $M$ and $M / J M$ is semisimple.

In $\S 3$, we define essentially finitely related perfect (EFR-perfect) modules. We prove that if the singular submodule of $R / J$ is zero, then a projective $R$-module is EFR-perfect if and only if it is FRperfect.

1. Preliminaries. This section contains properties fo projective covers needed for our purpose. Proposition 1.7 is an important step for proving our main theorem. This proposition may have its own value. In a later part of this section we mention some results about regular modules (R. Ware [8]) which will be used in $\S 2$.

Proposition 1.1 (Exercise 15(2), P. 203 [1]). Let $A, B$ be two $R$-modules. If $A$ and $A \oplus B$ have projective covers, then $B$ also has a projective cover.

Since $R / J$ is Jacobson semisimple, only projective modules over $R / J$ can have projective covers. Hence

Proposition 1.2. If an $R / J$-module $N$ has a projective cover as an $R$-module, then $N$ is projective as an $R / J$ module.

Proposition 1.3. Let $N$ be a small submodule of an $R$-module $M$. Let $U$ be any submodule of $M$. Then $M / U$ has a projective cover if and only if $M /(U+N)$ has a projective cover.

Proof. Since the natural map $M / U \rightarrow M /(U+N)$ is minimal, the proposition follows.

Proposition 1.4. Let $A=\bigoplus_{s \in S} A_{s}$ be an $R$-module such that $f_{s}: P_{s} \rightarrow A_{s}$ is a projective cover of $A_{s}$ for every $s \in S$. If $h: P \rightarrow A$ is a projective cover of $A$, then there exists an isomorphism $g: \oplus P_{s} \rightarrow P$ such that $(h \circ g) \mid P_{s}=f_{s}$.

Proof. Define $f: \oplus P_{s} \rightarrow \oplus A_{s}$ by $f \mid P_{s}=f_{s}$ for every $s \in S$. As $\oplus P_{s}$ is projective, there exists a homomorphism $g: \oplus P_{s} \rightarrow P$ such that $h \circ g=f$. As $h$ is a minimal epimorphism and $f$ is onto, $g$ is 
onto. As $P$ is projective, $g$ splits and $\operatorname{ker}(g)$ is a direct summand of $\oplus P_{s}$. Now $\operatorname{ker}(g) \subseteq \operatorname{ker}(f)=\bigoplus \operatorname{ker}\left(f_{\mathrm{s}}\right) \subseteq \bigoplus J P_{s} \subseteq J\left(\oplus P_{\mathrm{s}}\right)$; since $J\left(\oplus P_{s}\right)$ cannot contain a direct summand of $\oplus P_{s}, \operatorname{ker}(g)=0$.

Proposition 1.5 (cf. Nicholson [5, Lemma 1.6]). Let $N$ be a direct summand of a projective module $P$. Let $M^{\prime}$ be a submodule of $P$ such that $P=N+M^{\prime}$. Then $P=N \oplus M$ for some submodule $M \subseteq M^{\prime}$.

Corollary 1.6. Let $M$ be any projective module. Then decompositions modulo JM can be lifted if and only if for any direct summand $D$ of $M / J M$ there is a direct summand $B$ of $M$ such that $f(B)=D$, where $f: M \rightarrow M / J M$ is the natural map.

Proof. The only if part is trivial. Assume that the condition is satisfied. Suppose $M / J M=C \oplus D$. Let $B$ be a direct summand of $M$ such that $f(B)=D$. Now clearly $M=f^{-1}(C)+B$. Then by Proposition 1.5, we can write $M=A \oplus B$ where $A \subseteq f^{-1}(C)$. Then $M / J M=f(A)+D$. But $f(A) \subseteq f\left(f^{-1}(C)\right) \subseteq C$. Since $M / J M=C \oplus D$, $f(A)=C$. This completes the proof.

The following proposition is an important step towards proving the main theorem. It may also be of some independent interest.

Proposition 1.7. Let $M$ be a projective $R$-module, $f: M \rightarrow M / J M$ be the natural map and $A$ be any submodule of $M$. Assume either (a) $A$ is finitely generated or (b) $J M$ is small in $M$. Then the following conditions are equivalent:

(1) $M / A$ has a projective cover.

(2) $M=C \oplus T$ such that $C \subseteq A$ and $A \cap T$ is small in $M$.

(3) $f(A)$ is a summand of $M / J M$ and $f(A)$ has a projective cover as an $R$-module.

(4) There is a summand $C$ of $M$ (which is finitely generated if $A$ is finitely generated) such that $f(C)=f(A)$.

(5) $f(A)$ is a summand of $M / J M$ and for any decomposition $M / J M=f(A) \oplus B$ there is a decomposition $M=C \oplus D$ such that $f(C)=f(A), f(D)=B$ (where $C$ is finitely generated if $A$ is finitely generated).

Proof. The equivalence of conditions (1) and (2) is well known without (a) or (b). We prove $(2) \Rightarrow(3) \Rightarrow(4) \Rightarrow(5) \Rightarrow(1)$.

$(2) \Rightarrow(3)$. Since $M=C \oplus T, M / J M=f(C) \oplus f(T) . \quad C \cong A$ implies $A=C \oplus(A \cap T)$. This together with $A \cap T$ is small in $M$ shows that $f(A)=f(C)$. Hence $f(A)$ is a summand of $M / J M$. Consider the epimorphism $g=f \mid C: C \rightarrow f(A) ; \operatorname{ker}(g)=C \cap J M=J C$, since $C$ 
is direct summand of $M$. If $A$ is finitely generated or $J M$ is small in $M$, then $J C$ is small in $C$. In any case, $g: C \rightarrow f(A)$ is a projective cover.

$(3) \Rightarrow(4)$. Let $M / J M=f(A) \oplus T$ and $g: M / J M \rightarrow f(A)$ be the corresponding projection map. Then $g \circ f: M \rightarrow f(A)$ is an epimorphism and further $g \circ f(A)=f(A)$. Let $h: P \rightarrow f(A)$ be a projective cover. Then there exists $h^{\prime}: M \rightarrow P$ such that $g \circ f=h \circ h^{\prime}$. Since $f(A)=$ $g \circ f(A)$ and $h$ is minimal $h^{\prime}(A)=P$. This means $M=\operatorname{ker} h^{\prime}+A$ and ker $h^{\prime}$ is a summand of $M$, and so $M=\operatorname{ker} h^{\prime} \oplus A^{\prime}$ with $A^{\prime} \subseteq A$ by Proposition 1.5. Hence there is a splitting monomorphism $j: P \rightarrow M$ such that $j(P) \subseteq A$. Let $C=j(P)$. Then $(g \circ f) \mid C: C \rightarrow f(A)$ is a projective cover. Then clearly $f(C)=f(A)$. If $A$ is finitely generated, then so is $f(A)$ and consequently its projective cover $C$ is finitely generated.

$(4) \Rightarrow(5)$. Assume (4). $f(A)=f(C)$ is a direct summand of $M / J M$, since $C$ is a direct summd of $M$. Let $M / J M=f(A) \oplus B$ be any decomposition. We have $M / J M=f(C) \oplus B$; consequently $M=$ $C+f^{-1}(B)$. By Proposition 1.5, $M=C \oplus D$, where $D \subseteq f^{-1}(B)$. Clearly this is the required decomposition.

( 5$) \Rightarrow(1)$. Assume (5). Write $M=C \oplus S$ such that $f(C)=f(A)$. Let $\left\{a_{t}\right\}$ be a set of generators for $A$. Write $a_{t}=c_{t}+s_{t}, c_{t} \in C, s_{t} \in S$ for each $t$. Then $f\left(a_{t}\right)=f\left(c_{t}\right)+f\left(s_{t}\right)$. But $M / J M=f(C) \oplus f(S)$ and $f(A)=f(C)$ together imply $f\left(s_{t}\right)=$ zero i.e., $s_{t} \in J M$. Let $C^{\prime}=\Sigma R c_{t}$, We have

$$
f\left(C^{\prime}\right)=f\left(\Sigma R c_{t}\right)=\Sigma f\left(R c_{t}\right)=\Sigma f\left(R a_{t}\right)=f\left(\Sigma R a_{t}\right)=f(A)=f(C) .
$$

Consequently, $C^{\prime}+J M=C+J M$. Since $C$ is direct summand of $M, C^{\prime}+J C=C$. If $A$ is finitely generated so is $C$ by assumption and therefore $J C$ is small in $C$. If $J M$ is small in $M$, then $J C$ is small in $C$. In any case we have $C^{\prime}=C$. From $a_{t}=$ $c_{t}+s_{t}$ for each $t$ we see that $A+S^{\prime}=C^{\prime}+S^{\prime}=C+S^{\prime}$ where $S^{\prime}=$ $\Sigma R s_{t}$. Since each $s_{t} \in J M$, we see that $S^{\prime}$ is small in $M$ (if the indexing set of $t$ is finite or $J M$ small in $M$ ). By repeated use of Proposition 1.3 , we see that $M / A$ has a projective cover if and only if $M /\left(A+S^{\prime}\right)=$ $M /\left(C+S^{\prime}\right)$ has a projective cover. This is so if and only if $M / C \cong$ $S$ has a projective cover. But $S$ is itself projective. This completes the proof.

A module $M$ is called regular if $M$ is projective and every cyclic submodule of $M$ is a direct summand of $M$ (R. Ware [8, Definition 2.3]). R. Ware has proved that if $M$ is a regular $R$-module, then every finitely generated submodule of $M$ is a direct summand [8, Proposition 2.1] and $\bigoplus_{s} P_{s}$ is regular if and only if each $P_{s}$ is regular [8, Page 239].

We need the following propositions to prove our main theorem. 
Proposition 1.8 (R. Ware [8, Theorem 2.12]). An R-module $P$ is regular if and only if $P$ is isomorphic to a direct sum of principal left ideals of $R$, each of which is a regular module.

Proposition 1.9. Let $U$ be a direct summand of $\bigoplus_{s \in S} M_{s}$, where each $M_{s}$ is a regular $R$-module. Then $U \cong \bigoplus_{t \in T} R e_{t}$ where each $R e_{t}$ is a direct summand of $M_{s}$ for some $s \in S$.

Proof. By Proposition 1.8 it is enough to prove the result when $U$ is cyclic and hence when the set $S$ is finite. If the cardinality of $S$ is 1 , the result is obvious. Assume the result whenever the cardinality of $S \leqq n-1$. Let $S=\left\{s_{1}, \cdots, s_{n}\right\}$ and $U$ be a direct summand of $\bigoplus_{s \in S} M_{s}$. Suppose $f: M_{s_{1}} \oplus \cdots \oplus M_{s_{n}} \rightarrow M_{s_{n}}$ is the natural projection and $g=f \mid U$. As $g(U)$ is a direct summand of $M_{s_{n}}$, it is projective and $U \cong \operatorname{ker}(g) \oplus g(U)$. Since $\operatorname{ker}(g)$ is a direct summand of $M_{s_{1}} \oplus \cdots \oplus M_{s_{n-1}}$, the result follows from the induction hypothesis.

2. FR-Perfect modules. In this section we define the concept of FR-perfect modules and obtain our main theorem. Using this some characterizations of semiperfect rings and modules are obtained.

A module $N$ is said to be $M$-finitely related ( $M$-FR) if $N \cong M^{n} / B$, where $B$ is finitely generated. A module $N$ is called $M$-finitely presented $(M$-FP) if $N \cong P / U$, where $P$ is a direct sum of copies of $M$ and $U$ is a finitely generated submodule of $P$. A module $M$ is called FR-perfect (FP-perfect) if every $M$-FR( $M$-FP) module has a projective cover. $M$ is called semiperfect if it is projective and every factor module of $M$ has a projective cover.

THeOREm 2.1. Let $R$ be a ring and $M$ be any projective $R$-module. Let $f: M \rightarrow M / J M$ be the natural map. Then the following are equivalent.

(i) $M / J M$ is a regular $R / J$-module and for every direct decomposition $A \oplus B$ of $M / J M$, where $A$ is cyclic, there exists a decomposition $M=C \oplus D$, where $f(C)=A, f(D)=B$ and $C$ is cyclic.

(ii) $M / J M$ is a regular $R / J$-module and for every direct decomposition $A \oplus B$ of $M / J M$, where $A$ is finitely generated, there exists a direct decomposition $M=C \oplus D$, where $f(C)=A, f(D)=B$ and $C$ is finitely generated.

(iii) $M / J M$ is a regular $R / J$-module and every cyclic (finitely generated) submodule of $M / J M$ has a projective cover as an $R$-module.

(iv) $M$ is FR-perfect.

(v) $M / U$ has a projective cover for ever finitely generated submodule $U$ of $M$. 

of $M$.

(vi) $M / U$ has a projective cover for every cyclic submodule $U$

Proof. (i) $\Rightarrow$ (iii). Let $A$ be a cyclic submodule of $M / J M$. Since $M / J M$ is regular, we can write $M / J M=A \oplus B$. By (i) $M=C \oplus D$ where $f(C)=A$. Since $M / C \cong D$ is projective, by $(1) \Rightarrow(3)$ of Proposition 1.7, we see that $A=f(C)$ has a projective cover. Since any finitely generated submodule of $M / J M$ is a finite direct sum of cyclic submodules of $M / J M$, we get the result.

(iii) $\Rightarrow$ (iv). In view of Proposition 1.9 any direct sum of copies of $M$ also satisfies condition (iiii). Thus it is enough to prove that $M / U$ has a projective cover whenever $U$ is finitely generated. But this is clear in view of $(3) \Rightarrow(1)$ of Proposition 1.7.

(iv) $\Rightarrow$ (v) is obvious.

(v) $\Rightarrow$ (ii). Using $(1) \Rightarrow(5)$ of Proposition 1.7 we get this.

(ii) $\Rightarrow$ (vi). Using (5) $\Rightarrow(1)$ of Proposition 1.7 we get this.

$(v i) \Rightarrow(i)$. This follows from $(1) \Rightarrow(5)$ of Proposition 1.7 and the fact that the projective cover of a cyclic module is cyclic.

Corollary 2.2. Let $M$ be a projective module such that $J M$ is small in $M$. Let $f: M \rightarrow M / J M$ be the natural map. Then the following are equivalent.

(a) $M$ is FR-perfect.

(b) $M / J M$ is a regular $R / J$-module and any direct decomposition of $M / J M$ can be lifted to a direct decomposition of $M$.

(c) $M / U$ has a projective cover whenever $f(U)$ is finitely generated.

(d) $M / J M$ is a regular $R / J$-module and every direct summand of $M / J M$ has a projective cover as an $R$-module.

Proof. $\quad(\mathrm{a}) \Rightarrow(\mathrm{b}) . \quad$ As $M / J M$ is a regular $R / J$-module it is enough to prove that a direct decomposition $\bigoplus_{s \in S} V_{s}$ of $M / J M$, where each $V_{s}$ is cyclic, can be lifted up. Let $f_{s}: P_{s}-V_{s}$ be projective covers for every $s \in S$. Since $J M$ is small in $M$, by Proposition 1.4, $M=\oplus P_{s}^{\prime}$, where $f\left(P_{s}^{\prime}\right)=V_{s}$ for every $s \in S$.

(b) $\Rightarrow($ c). Let $U \subseteq M$ be a submodule such that $f(U)$ is finitely generated. Then $f(U)$ is a direct summand of $M / J M$. By assumption we can write $M=C \oplus T$ such that $f(C)=f(U)$. Then since $J M$ is small in $M$, by $(4) \Rightarrow(1)$ of Proposition $1.7, M / U$ has a projective cover.

(c) $\Rightarrow$ (a) is obvious in view of Theorem 2.1.

(b) $\Rightarrow(\mathrm{d})$. Let $M / J M=V \oplus W$. Then there exits a decomposition $A \oplus B$ of $M$ such that $f(A)=V$ and $f(B)=W$. Let $g=f \mid A$. Then $\operatorname{ker}(g)=J A$ is small in $A$ since $J M$ is small in $M$. So $g: A \rightarrow V$ is a projective cover of $V$. 
$(\mathrm{d}) \Rightarrow(\mathrm{a})$. This is clear by Theorem 2.1 (iii) $\Rightarrow$ (iv).

CoROLlaRY 2.3. Let $M$ be a projective $R$-module and $J M$ be small in $M$. If $M$ is FR-perfect then $M \cong \bigoplus_{s \in S} R e_{s}$, where each $e_{s}$ is an idempotent in $R$.

Proposition 2.4. Let $M=\bigoplus_{s \in S} M_{s}$, be a a projective $R$-module. $M$ is FR-perfect if and only if each $M_{s}$ is FR-perfect.

Proof. The only if part is obvious. For the converse, it is sufficient to prove for finite direct sums in view of Theorem 2.1 (iii). This can be easily seen by using Proposition 1.9 and Theorem 2.1 (iii).

COROLLARY 2.5. A projective module is FR-perfect if and only if it is FP-perfect.

Remark. W. K. Nicholson [5, Page 1107] calls a module $M$ semiregular if for every $m \in M$, there exists a decomposition $M=$ $P \oplus Q$ where $P \subseteq R m, P$ is projective and $R m \cap Q$ is small in $M$. If $M$ is projective, then $M$ is semiregular if and only if $M / R m$ has a projective cover for every $m \in M$. By Theorem 2.1, a projective module is semiregular if and only if $M$ is FR-perfect. Theorem 2.1 (ii) $\Leftrightarrow$ (iv) is an improvement of Proposition 1.17 of [5]. We do not know whether if $M$ is a projective $R$-module and $M / J M$ is a finitely generated $R / J$-regular module, then $M$ is finitely generated. If this is so then the finitely generated condition on $C$ in Theorem 2.1 (ii) can be removed. Also Proposition 1.17 of [5] will be then be true without $J M$ being small in $M$. For a projective module $M$ to be FR-perfect, $J M$ need not be small in $M$. Let $R$ be a ring which is semiperfect but not perfect. Then $M$, a countable direct sum of copies of $R$, is FR-perfect but $J M$ is not small in $M$. We do not know whether every FR-perfect projective module is a direct sum of cyclics.

A module $Q$ is called Quasi-projective if for every exact sequence $Q \rightarrow A \rightarrow 0$, the induced sequence $\operatorname{Hom}(Q, Q) \rightarrow \operatorname{Hom}(Q, A) \rightarrow 0$ is exact. Let $M$ be an $R$-module. A minimal epimorphism $f: Q \rightarrow M$ is called a quasi-projective cover of $M$ if $Q$ is quasi-projective and $Q / T$ is not quasi-projective whenever $T \subseteq \operatorname{ker}(f)$.

Lemma 2.6 ([6, Lemma 3.2]). A module $A$ is projective if and only if there exists an epimorphism $P \rightarrow A$ with $P$ projective and $A \oplus P$ quasi-projective.

Lemma 2.7. If $P \oplus A$ has a quasi-projective cover where $P$ is 
projective and $A$ is an epimorphic image of $P$, then $A$ has a projective cover.

Proof. Let $g: Q \rightarrow P \oplus A$ be a quasi-projective cover. As $P$ is projective $Q$ splits. We can write $Q=P_{1} \oplus g^{-1}(A)$ where $P_{1} \cong P$. Then there is an epimorphism $f: P_{1} \rightarrow A$. We note that $g \mid g^{-1}(A)$ : $g^{-1}(A) \rightarrow A$ is a minimal epimorphism. Hence there is an epimorphism from $P_{1}$ onto $g^{-1}(A)$. Then Lemma 2.6 shows that $g^{-1}(A)$ is projective. Hence $A$ has a projective cover.

COROLlaRy 2.8. For a projective module $M$ conditions in Theorem 2.1 are equivalent to:

(vii) every M-FR module has a quasi-projective cover.

(viii) $(M / U) \oplus M$ has a quasi-projective cover whenever $U$ is finitely generated.

( ix ) $(M / U) \oplus M$ has a quasi-projective cover whenever $U$ is cyclic.

In particular by taking $M=R$ we get the following result. The equivalence of (1) and (2) is already proved in [7, Proposition 5].

COROLlary 2.9. The following are equivalent for any ring $R$

(i) $R$ is left FR-perfect.

(ii) $R / J$ is Von-Neumann regular and idempotents can be lifted modulo $J$.

(iii) $R / J$ is Von-Neumann regular and every cyclic (finitely generated) left ideal of $R / J$ has a projective cover as an $R$-module.

(iv) $R / U$ has a projective cover whenever $U$ is finitely generated left ideal of $R$.

(v) $R / U$ has a projective cover whenever $U$ is a principal left ideal of $R$.

(vi) $R$ is right FR-perfect.

(vii) $R$ is right (left) FR-quasi-perfect.

Theorem 19.27 of [3] tells us that there exists plenty of FRperfect rings. We rephrase it in our notation.

Proposition 2.10 (C. Faith [3, Theorem 19.27]). If $Q$ is a quasiinjective module, then the endomorphism ring of $Q$ is self injective FR-perfect ring.

COROLLARY 2.11. Any self injective ring is a FR-perfect ring.

From the proof of Theorem 5.6 of [4] we get, 
THEOREM 2.12. If $M$ is an FR-perfect module and if $P$ is a projective cover of $M$, then $P$ is FR-perfect.

A factor module of an FR-perfect module need not be FR-perfect. Let $R$ be a Von-Neumann regular ring which is not semisimple. Then $R$ as a module over itself is an example. But if $M$ is an FRperfect module, then $M / U$ is FR-perfect whenever $U$ is finitely generated.

E. A. Mares [4, Theorems 3.3, 3.5, 4.3 and 5.1] has proved that a projective module $M$ is semiperfect if and only if it has the following properties (1) $M / J M$ is semisimple (that is every submodule is a summand), (2) Every direct decomposition of $M / J M$ can be lifted to a direct decomposition of $M$ and (3) $J M$ is small in $M$. We give below some characterizations of a projective semiperfect module.

It can be seen from Proposition 1.7 that a projective module $M$ is semiperfect if and only if $J M$ is small in $M, M / J M$ is semisimple and every submodule of $M / J M$ has a projective cover. From Corollary 2.2 we get

Proposition 2.13. A projective module $M$ is semiperfect if and only if it is FR-perfect, $J M$ is small in $M$ and $M / J M$ is semisimple.

A module is called finite dimensional if it contains no infinite direct sum of nonzero submodules.

THEOREM 2.14. Let $M$ be a projective FR-perfect module. If $M$ is finite dimensional, then $M$ is semiperfect.

Proof. Let $U$ be a submodule of $M / J M$. We show that $U$ is finitely generated. Suppose not. Let $m_{1} \in U$. Then $M / J M=R m_{1} \oplus$ $N_{1}$ and this decomposition be lifted to a decomposition $M=T_{1} \oplus L_{1}$. There exists $m_{2} \in U$ such that $m_{2} \notin R m_{1}$. Let $m_{2}=r m_{1}+n_{2}, n_{2} \in N_{1}$. Then $N_{1}=L_{1} / J L_{1}=R n_{2} \oplus N_{2}$ and this decomposition can be lifted to a decomposition $L_{1}=T_{2} \oplus L_{2}$. Hence if $U$ is not finitely generated, then $M$ is not finite dimensional. Thus $M / J M$ is a Noetherian and regular module and so is a finite direct sum of simple modules. Hence $M$ is a semiperfect.

THeOREM 2.15. A projective module $M$ is semiperfect if and only if $M / U$ has a projective cover for any countably generated submodule $U$ of $M$ and $J M$ is small in $M$.

Proof. The 'only if' part follows from Mares' result [4, Theorem 3.3]. Conversely suppose that the conditions are satisfied. By 
Theorem 2.1, (v) $\Rightarrow$ (iv), $M$ is FR-perfect. Then by Corollary 2.3, $M$ is a direct sum of cyclics. Clearly any direct summand of $M$ also satisfies the conditions. Hence by [4, Theorem 5.2], it is enough to prove the result when $M$ is cyclic. Let $f: M \rightarrow M / J M$ be the natural map. Let $A$ be a countably generated submodule of $M / J M$ and let $U$ be a countably generated submodule of $M$ such that $f(U)=A$. Since $M / U$ has a projective cover, $M=K \oplus P$, where $K \cong U$ and $P \cap U$ is small in $M$. As $K$ is cyclic, $A=f(U)=f(K)$ is cyclic. Hence $M / J M$ is Noetherian. As $M / J M$ is regular, it is semisimple. Using Corollary 2.13 we see that $M$ is semiperfect.

CoROllaRy 2.16 (Nicholson [5, Corollary 2.10]). Let $R$ be a ring. Then $R$ is semiperfect if and only if $R / U$ has a projective cover whenever $U$ is a countably generated left ideal.

Let $R$ be a ring which has ascending chain condition on left ideals $R e, e^{2}=e$. It is easy to see that such a $\operatorname{ring} R$ also satisfies descending chain condition on left ideals $R e, e^{2}=e$. We show that for such a ring $R$ any projective FR-perfect module $M$ is semiperfect if and only if $J M$ is small in $M$.

THEOREM 2.17. Let $R$ be a ring with ascending chain condition on left ideals $R e, e^{2}=e$. Then a projective FR-perfect module is semiperfect if and only if $J M$ is small in $M$.

Proof. Let $A$ be a cyclic summand of $M / J M$. Then $M=C \oplus D$, $f \mid C: C \rightarrow C / J C=A$ is a projective cover, where $f: M \rightarrow M / J M$ is the natural map. As $A$ is cyclic, $C$ is cyclic and hence $C \cong R e, e^{2}=e$. From the given condition on $R$ we see that $C$ is a finite direct sum of indecomposable modules. Let $C=\bigoplus_{i=1}^{k} C_{i}$, where each $C_{i}$ is a indecomposable cyclic projective module. Then

$$
A=\bigoplus_{i=1}^{k} A_{i}, f\left(C_{i}\right)=C_{i} / J C_{i}=A_{i} .
$$

As $A_{i}$ is regular, if it is not simple, then $A_{i}$ is decomposable. As any direct decomposition of $A_{i}$ can be lifted to a direct decomposition of $C_{i}$, this would imply that $C_{i}$ is decomposable. Hence $A$ is a direct sum of simple modules. As $M / J M$ is a regular module, it is a semisimple module. If $J M$ is small in $M$, then Proposition 2.13 shows that $M$ is semiperfect.

CoROLLARY 2.18. A ring $R$ is semiperfect if and only if $R$ is FR-perfect and $R$ satisfies ascending chain condition on left ideals $R e, e^{2}=e$. 
THeOREM 2.19. Let $M=\bigoplus_{s \in S} M_{s}$ be a FR-projective module. If $P$ is a direct summand of $M$ such that $J P$ is small in $P$, then $P$ is a direct sum of cyclic submodules which are isomorphic to direct summands of the modules $M_{s}, s \in S$.

Proof. Let $f: M \rightarrow M / J M$ be the natural map. By Proposition 1.9 , we see that $f(P) \cong \bigoplus_{i \in I} Q_{i}$, where each $Q_{i}$ is a cyclic summand of $f\left(M_{s_{i}}\right)$ for some $s_{i} \in S$. Since $M_{s_{i}}$ is FR-perfect projective there exists a cyclic summand $P_{i}$ of $M_{s_{i}}$ such that $f\left(P_{i}\right)=Q_{i}$ [Theorem 2.1, (i)]. For each $i, f \mid P_{i}: P_{i} \rightarrow Q_{i}$ is a projective cover. As $J P$ is small in $P$, by using Corollary 1.4 , it is easy to see that $P \cong \bigoplus P_{i}$ (external direct sum).

As any semiperfect module is a direct sum of indecomposables [4, Corollary 4.4], we get the following theorem of E. A. Mares as a corollary.

Corollary 2.20 (E. A. Mares [4, Theorem 5.5]). Let $P$ be a direct summand in a direct sum of semiperfect modules, $F=$ $\bigoplus_{i \in I} M_{i}=P \oplus Q$, and let JP be small in $P$; then $P$ is a direct sum of indecompasable submodules which are isomorphie to direct summands of $M_{i}, i \in I$.

3. EFR-Perfect modules. In this section we generalize the concept of FR-perfect modules. A submodule $U$ of a module $M$ is called essential in $M$ if $K \cap U=0$ implies $K=0$ for any submodule $K$ of $M$. A module $U$ is called essentially finitely generated (EFG) if it contains a finitely generated essential submodule. A module $N$ is called $M$-essentially finitely related ( $M$-EFR) if $N \cong M^{n} / U$, where $U$ is an EFG submodule of $M^{n}$. A module $M$ is called EFR-perfect if every $M$-EFR module has a projective cover.

The following proposition gives a characterization of EFR-perfect modules.

Proposition 3.1. Let $M$ be a projective $R$-module such that JM is small in $M$. Then the following conditions are equivalent

(i) $M$ is EFR-perfect.

(ii) $M$ is FR-perfect and for every EFG submodule $U$ of $M^{n}$, $\left(U+J M^{n}\right) / J M^{n}$ is finitely generated for every $n$.

(iii) $M$ is EFR-quasi-perfect.

Proof. The equivalence of (i) and (iii) follows from Lemma 2.7.

(ii) $\Rightarrow$ (i ). Since $M^{n}$ also satisfies condition (ii) for every integer $n$, it is enough to prove $M / U$ has a projective cover whenever $U$ is an EFG submodule of $M$. By Corollary $2.2, M / U$ has a projective cover. 
(i) $\Rightarrow$ (ii). Let $U$ be an EFG submodule of $M^{n}$. Let $f_{n}: M^{n} \rightarrow$ $M^{n} / J M^{n}$ be the natural map. As $M^{n} / U$ has a projective cover, $M^{n}=K \oplus T$ where $K \subseteq U$ and $U \cap T$ is small in $M^{n}$. Suppose $f_{n}(K)=f_{n}(U)$ is infinitely generated. Then $f_{n}(K)$ is an infinite direct sum of cyclics and hence by Proposition 1.4, $K$ is an infinite direct sum of cyclics and $U=K \oplus(U \cap T)$. So $U$ cannot be an EFG module.

If $M$ is an $R$-module, $Z(M)=$ the singular submodule of $M=$ $\left\{m \in M \mid \operatorname{ann}_{R}(m)\right.$ is essential in $\left.R\right\}$.

Lemma 3.2 [2, Proposition 1.1(v)]. Let $A$ and $B$ two $R$-modules. If $f: A \rightarrow B$ is an epimorphism, $Z_{R}(B)=0$ and $C$ is essential submodule of $A$, then $f(C)$ is essential in $B$.

Proposition 3.3. Let $R$ be a ring such that $Z(R / J)=0$. Then a projective $R$-module $M$ is EFR-perfect if and only if it is FRperfect.

Proof. Any EFG submodule of a regular module is finitely generated. If $U$ is an EFG submodule of $M$, then $(U+J M) / J M$ is an EFG submodule of $M / J M$.

COROLlaRY 3.4. Let $R$ be a Von-Neumann regular ring. A projective $R$-module $M$ is FR-perfect if and only if it is EFRperfect.

\section{REFERENCES}

1. F. W. Anderson and K. R. Fuller, Rings and Categories of Modules, SpringerVerlag, 1974.

2. V. C. Cateforis, Flat regular rings, Trans. Amer. Math. Soc., 138 (1969), 241-249.

3. C. Faith, Algebra II-Ring Theory, Springer-Verlag, 1976.

4. E. A. Mares, Semi-perfect modules, Math. Zeitschr, 82 (1963), 347-360.

5. W. K. Nichoison, Simiregular modules and rings, Canad. J. Math., 28 (1976), 1105-1120.

6. K. M. Rangaswami and N. Vanaja, Quasi projectives in abelian categories, Pacific J. Math., 43 (1972), 221-238.

7. K. M. Rangaswami and N. Vanaja, Rings over which finitely related by torsion modules have projective covers, Communications in Algebra, 2 (1974), 351-363.

8. R. Ware, Endomorphism rings of projective modules, Trans. Amer. Math. Soc., 155 (1971), 253-266.

Received May 8, 1979 and in revised form April 29, 1980.

UNIVERSITY OF BOMBAY,

Bombay 400 098, India 


\section{PACIFIC JOURNAL OF MATHEMATICS}

\section{EDITORS}

DONALD BABBITT (Managing Editor)

University of California

Los Angeles, California 90024

Hugo RossI

University of Utah

Salt Lake City, UT 84112

C. C. MOORE and ANDREW OGG

University of California

Berkeley, CA 94720

\section{J. DugundJI}

Department of Mathematics University of Southern California Los Angeles, California 90007

R. FinN and J. Milgram Stanford University Stanford, California 94305

\section{ASSOCIATE EDITORS}
R. ARENS
E. F. BECKENBACH
B. H. Neumann
F. WOLF
K. YoshidA

\section{SUPPORTING INSTITUTIONS}

UNIVERSITY OF ARIZONA

UNIVERSITY OF BRITISH COLUMBIA

CALIFORNIA INSTITUTE OF TECHNOLOGY

UNIVERSITY OF CALIFORNIA

MONTANA STATE UNIVERSITY

UNIVERSITY OF NEVADA, RENO

NEW MEXICO STATE UNIVERSITY

OREGON STATE UNIVERSITY
UNIVERSITY OF OREGON

UNIVERSITY OF SOUTHERN CALIFORNIA

STANFORD UNIVERSITY

UNIVERSITY OF HAWAII

UNIVERSITY OF TOKYO

UNIVERSITY OF UTAH

WASHINGTON STATE UNIVERSITY

UNIVERSITY OF WASHINGTON 


\section{Pacific Journal of Mathematics}

\section{Vol. 96, No. $1 \quad$ November, 1981}

Hédi Amara, Groupe des classes et unité fondamentale des extensions quadratiques relatives à un corps quadratique imaginaire principal $\ldots \ldots \ldots 1$

Douglas S. Bridges, On the isolation of zeroes of an analytic function $\ldots \ldots 13$ Andrew J. Casson and John L. Harer, Some homology lens spaces which

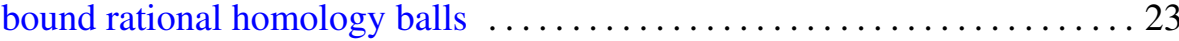

Z. A. Chanturia, On the absolute convergence of Fourier series of the classes $H^{\omega} \cap V[v]$

J.-F. Colombeau and Mário Carvalho Matos, On some spaces of entire functions defined on infinite-dimensional spaces $\ldots \ldots \ldots \ldots \ldots \ldots \ldots 63$

Edwin Duda, Pointwise periodic homeomorphisms on chainable continua . . .77

Richard F. Gustafson, A simple genus one knot with incompressible spanning surfaces of arbitrarily high genus $\ldots \ldots \ldots \ldots \ldots \ldots \ldots \ldots 1$

Fumio Hiai, Masanori Ohya and Makoto Tsukada, Sufficiency, KMS condition and relative entropy in von Neumann algebras

Ted Hurley, Intersections of terms of polycentral series of free groups and free Lie algebras. II .................................. 111

Robert Edward Jamison, II, Partition numbers for trees and ordered sets . . 115 R. D. Ketkar and N. Vanaja, A note on FR-perfect modules ............. 141 Michihiko Kikkawa, On Killing-Ricci forms of Lie triple algebras ....... 153

Jorge Lewowicz, Invariant manifolds for regular points 163

Richard W. Marsh, William H. Mills, Robert L. Ward, Howard Rumsey and Lloyd Richard Welch, Round trinomials .....

Claude Schochet, Topological methods for $C^{*}$-algebras. I. Spectral sequences

Yong Sian So, Polynomial near-fields?

Douglas Wayne Townsend, Imaginary values of meromorphic functions in the disk

Kiyoshi Watanabe, Coverings of a projective algebraic manifold .. 243

Martin Michael Zuckerman, Choosing $l$-element subsets of $n$-element sets 